\title{
Author Correction: Mitochondrial DNA repair and replication proteins revealed by targeted chemical probes
}

Simon Wisnovsky, Sae Rin Jean and Shana O Kelley

Correction to: Nature Chemical Biology https://doi.org/10.1038/nchembio.2102, published online 30 May 2016

In the version of this article initially published, Sanduni Liyanage and Aaron Schimmer were not properly acknowledged as co-authors. Both authors have now been included in the current author list, and their contributions are now specified in the author contributions statement. The error has been corrected in the PDF and HTML versions of this article.

\section{Publisher Correction: A robotic multidimensional directed evolution approach applied to fluorescent voltage reporters}

Kiryl D. Piatkevich, Erica E. Jung, Christoph Straub, Changyang Linghu, Demian Park, Ho-Jun Suk, Daniel R. Hochbaum (D, Daniel Goodwin, Eftychios Pnevmatikakis (D), Nikita Pak, Takashi Kawashima, Chao-Tsung Yang, Jeffrey L. Rhoades (D, Or Shemesh, Shoh Asano, Young-Gyu Yoon (D), Limor Freifeld (D), Jessica L. Saulnier, Clemens Riegler, Florian Engert, Thom Hughes (D, Mikhail Drobizhev, Balint Szabo, Misha B. Ahrens, Steven W. Flavell, Bernardo L. Sabatini (iD) and Edward S. Boyden

Correction to: Nature Chemical Biology https://doi.org/10.1038/s41589-018-0004-9; published online 26 February 2018.

In the version of this article originally published, the bottom of Figure 4f,g was partially truncated in the PDF. The error has been corrected in the PDF version of this article.

\section{Publisher Correction: Resistance to nonribosomal peptide antibiotics mediated by $D$-stereospecific peptidases}

Yong-Xin Li, Zheng Zhong (D), Peng Hou, Wei-Peng Zhang and Pei-Yuan Qian

Correction to: Nature Chemical Biology https://doi.org/10.1038/s41589-018-0009-4; published online 26 February 2018.

In the version of this article originally published, the links and files for the Supplementary Information, including Supplementary Tables 1-5, Supplementary Figures 1-25, Supplementary Note, Supplementary Datasets 1-4 and the Life Sciences Reporting Summary, were missing in the HTML. The error has been corrected in the HTML version of this article. 